\title{
Matching and transporting an intense ion beam through a solenoid focusing channel
}

\author{
J. E. Coleman \\ Department of Nuclear Engineering, University of California at Berkeley, 4155 Etcheverry Hall, MC 1730, \\ Berkeley, California 94720, USA, \\ and Lawrence Berkeley National Laboratory, 1 Cyclotron Road, Berkeley, California 94720, USA \\ P. A. Seidl, E. Henestroza, M. A. Leitner, P. K. Roy, W. L. Waldron, and D. Baca \\ Lawrence Berkeley National Laboratory, 1 Cyclotron Road, Berkeley, California 94720, USA \\ I. Haber \\ University of Maryland, College Park, Maryland 20742-3511, USA
}

A. W. Molvik and W. M. Sharp

Lawrence Livermore National Laboratory, Livermore, California 94550, USA

J. Armijo

Ecole Normale Superieure, Paris, France,

and Lawrence Berkeley National Laboratory, 1 Cyclotron Road, Berkeley, California 94720, USA

(Received 20 August 2007; published 6 May 2008)

\begin{abstract}
An experiment to inject and match a $10 \mu \mathrm{s}$, singly charged $\mathrm{K}^{+}$ion bunch at an ion energy of $0.3 \mathrm{MeV}$, current of $45 \mathrm{~mA}$, and dimensionless perveance of $10^{-3}$ into a solenoid lattice has been carried out at LBNL. The principal objective of this experiment is to match and transport the space-charge dominated ion beam and compare predicted and measured emittance. Initial investigation also presented the opportunity to study electron cloud effects and the effects of misalignments. A qualitative comparison of experimental and calculated results are presented, which include time resolved current density, transverse distributions, and phase space of the beam at different diagnostic planes.
\end{abstract}

DOI: 10.1103/PhysRevSTAB.11.050101

\section{INTRODUCTION}

Space-charge dominated ion beams of various masses and charge states can be used to drive fusion targets and heat targets to the warm dense matter (WDM) regime. Solenoids can focus axisymmetrically and transport more line charge density for low mass ions at low kinetic energies than magnetic quadrupoles [1,2]. Solenoids are used for low energy beam transport to match space-charge dominated hydrogen ions $\left(\mathrm{H}^{+}, \mathrm{H}_{2}{ }^{+}\right.$, and $\left.\mathrm{H}^{-}\right)$into radio frequency quadrupoles (RFQ) [3-6]. Although the space charge is significant, these beams interact with background gas at the ion source and undergo partial neutralization. The overall beam transmission from matching into the RFQ is typically $90 \%$. This partial neutralization also leads to overfocusing in the solenoids and emittance growth.

One design for a near-term ion accelerator capable of heating targets to $\mathrm{T}_{e}>1 \mathrm{eV}$ will have a 20-30 solenoid lattice. A high perveance, short pulse beam will be injected into a solenoid matching section. After matching the spacecharge dominated ion beam, it will be accelerated to provide the necessary kinetic energy (close to the Bragg peak) at the center of the target $[7,8]$.

The neutralized drift compression experiment (NDCX) at LBNL is exploring the physical limits of compression and focusing of ion beams for heating material to WDM and inertial fusion ignition conditions $[9,10]$. In prepara-
PACS numbers: 29.27.Bd, 34.35.+a, 41.85.Lc, 52.59.Sa

tion for NDCX, the solenoid transport experiment (STX) described herein is studying the matching and transport of a singly charged, $300-\mathrm{kV}, 45-\mathrm{mA}$ potassium ion beam. A solenoid field of $2.5 \mathrm{~T}$ is used to match the space-charge self-field of a beam. The beam current is lower than ultimately required, but the generalized perveance is high and characteristic of the conditions at the front end for these applications. The ion source is aluminosilicate which preferentially emits ions at $\mathrm{T} \sim 1000^{\circ} \mathrm{C}[11,12]$. This allows low-emittance ion extraction with negligible gas interaction. Low emittance is a priority in order to achieve the high intensity necessary for heating targets. Partial neutralization in the gun is negligible and greater than $99 \%$ of the beam injected into the solenoids is transmitted.

\section{HIGH CURRENT INJECTOR}

The beam used for the STX was accelerated through a 12-cm long diode and extracted through a 4-cm diameter aperture (Fig. 1) by a $300 \mathrm{kV}, 10-\mu$ s long voltage pulse (Fig. 2). Two cylindrical electron suppression electrodes, with a removable current reducing aperture between them, followed directly downstream. The fully extracted beam current $(45 \mathrm{~mA})$ measured with a $6.35 \mathrm{~cm}$ diameter Faraday cup displayed a relatively flat profile through the pulse duration (Fig. 2). Transverse beam dynamics measurements were made to characterize the injected beam 


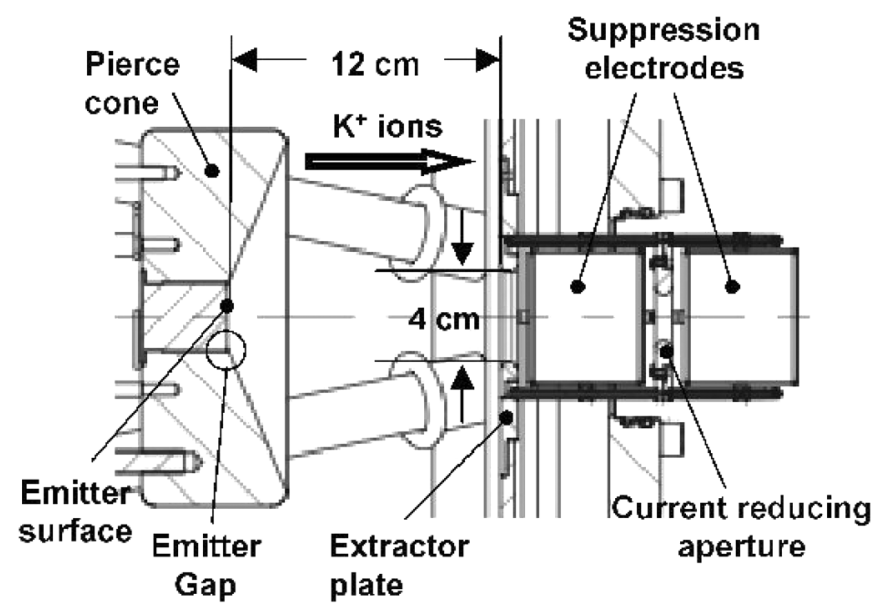

FIG. 1. Diagram of the Pierce diode geometry and suppression electrodes with removable aperture downstream (note aperture not installed for these experiments).

between 15 and $31 \mathrm{~cm}$ downstream of the exit of the diode before installing the solenoids.

Transverse phase-space measurements were made using two separate diagnostic techniques. One method used an

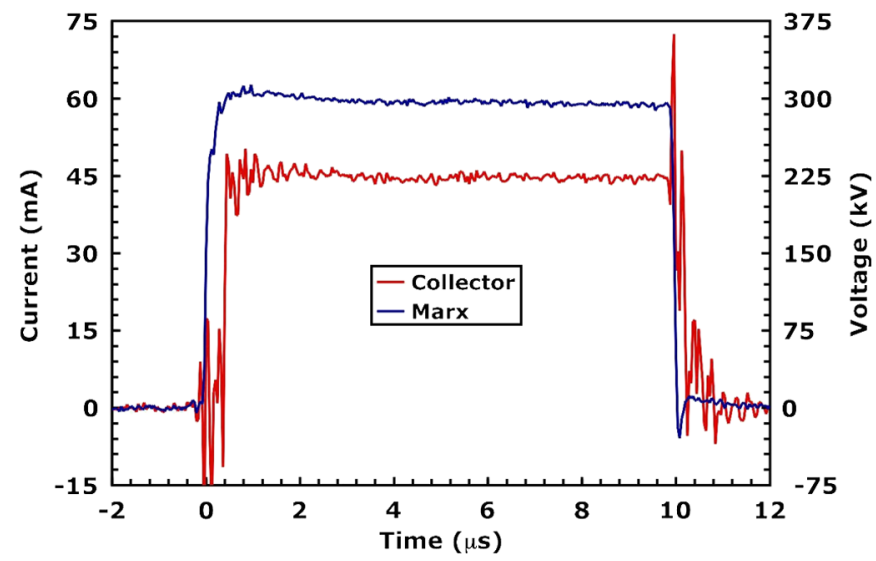

FIG. 2. (Color) Marx voltage waveform used to extract the beam through the diode (blue) and the measured beam current $28 \mathrm{~cm}$ downstream of the diode (red; $z=40 \mathrm{~cm}$ ).

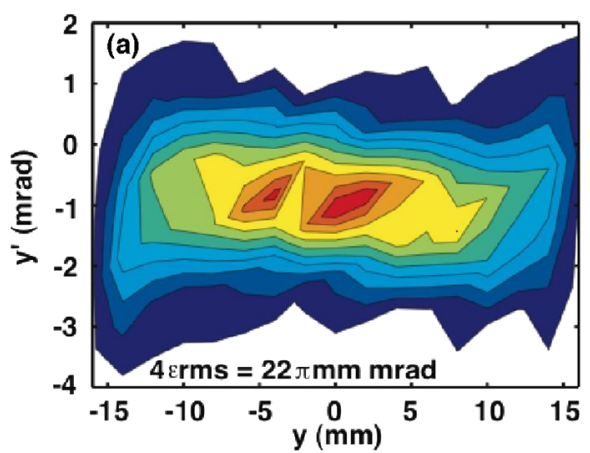

upstream slit and a downstream slit and a Faraday collector (slit cup) separated by $\sim 10 \mathrm{~cm}$. The slits used were $0.1 \mathrm{~mm} \times 57 \mathrm{~mm}$. Step sizes for these measurements were typically $1 \mathrm{~mm} \times 1 \mathrm{mrad}$. The slit cup generally measured tens of millivolts ( $\sim \mu \mathrm{A}$ of current) and the $\mathrm{S} / \mathrm{N}$ was close to 30 . The second method used a $100-\mu \mathrm{m}$ thick alumina scintillator and an image-intensified gatedCCD camera that imaged beam-induced light emission after masking the beam with a single upstream slit [13]. The measured distribution shown in Fig. 3 was made using the first technique. The distribution was nearly uniform and axisymmetric, although it had an angle offset of $1 \mathrm{mrad}$ relative to the desired beam line. The measured normalized emittance ( $\left.4 \epsilon_{\text {nrms }}=0.088 \pi \mathrm{mm} \mathrm{mrad}\right)$ was only a factor of 2 greater than the calculated thermal emittance for the ion source. The envelope parameters were also constant over the full pulse.

A third method was used to measure the transverse profile with a single slit and Faraday collector. The transverse beam distribution $[J(x, y)]$ was also measured at the scintillator plane. Because of the $45 \mathrm{mrad}$ divergence angle and the beam space charge, the measured $2 \mathrm{rms}$ radius of the beam expanded from $21 \mathrm{~mm}$ to $26 \mathrm{~mm}$ over the $6 \mathrm{~cm}$ drift distance from the slit-cup plane to the scintillator plane (Fig. 4). These measurements show a symmetric distribution when projected onto the $x$-axis, however the distribution in Fig. 4(b) has radial contours. There is a slight peak in the center, moving out radially the intensity falls until reaching a higher intensity rim around the edge of the beam, also most of the upper left region of the distribution is below 50\% of the peak intensity. These radial contours in the distribution are a sign of nonuniform focusing fields from the diode.

Particle in the cell simulations using the WARP code [14] were performed to understand how these contours in the distribution are generated. Studies of the Pierce geometry have indicated that the placement of the emitter surface flush to the knife edge of the Pierce cone (Fig. 1) is necessary for producing a uniform distribution without radial contours [Fig. 5(a)]. Calculations show that placing the emitter surface 22 mils back from the knife edge of the

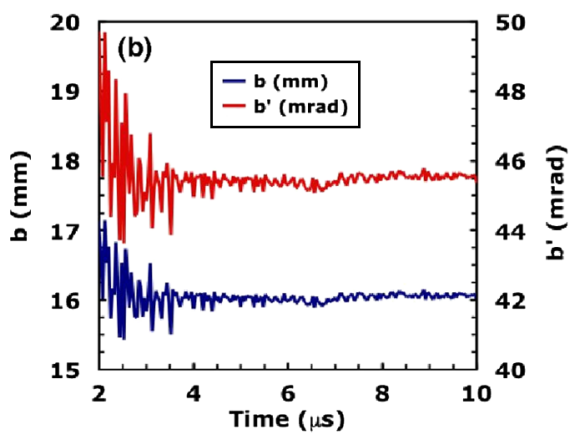

FIG. 3. (Color) (a) Sheared phase-space distribution integrated over a 500 ns gate in the middle of the $10 \mu \mathrm{s}$ beam pulse; (b) beam envelope as a function of time $15 \mathrm{~cm}$ downstream of the exit of the extractor $(z=27 \mathrm{~cm}$; no solenoids). 

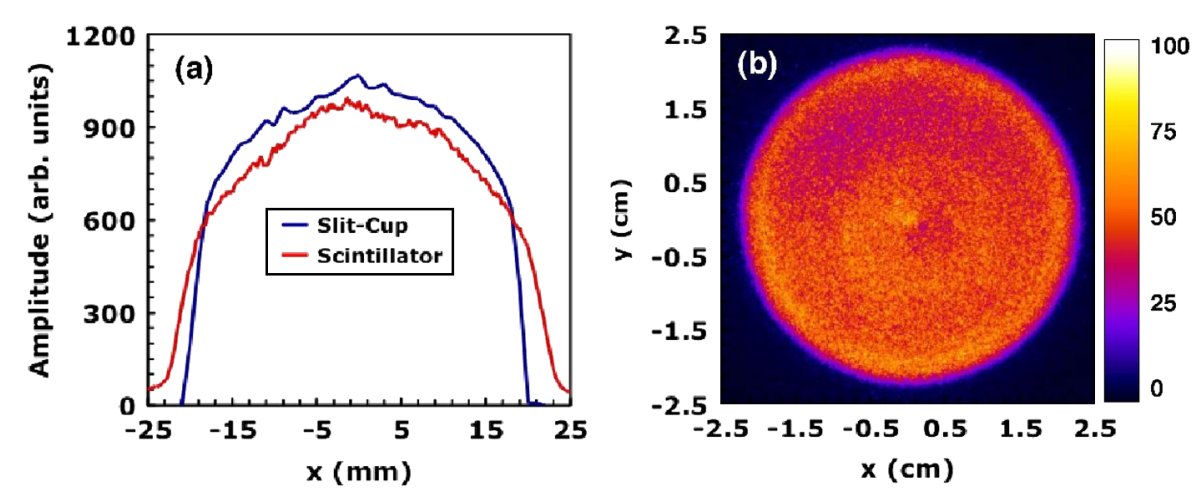

FIG. 4. (Color) Transverse profile of the beam measured: (a) $25 \mathrm{~cm}(z=37 \mathrm{~cm})$ and $31 \mathrm{~cm}(z=43 \mathrm{~cm})$ downstream of the exit of the extractor. (b) Transverse beam distribution measured at the scintillator plane. All integrated over a 500 ns gate in the middle of the $10 \mu \mathrm{s}$ beam pulse (no solenoids).
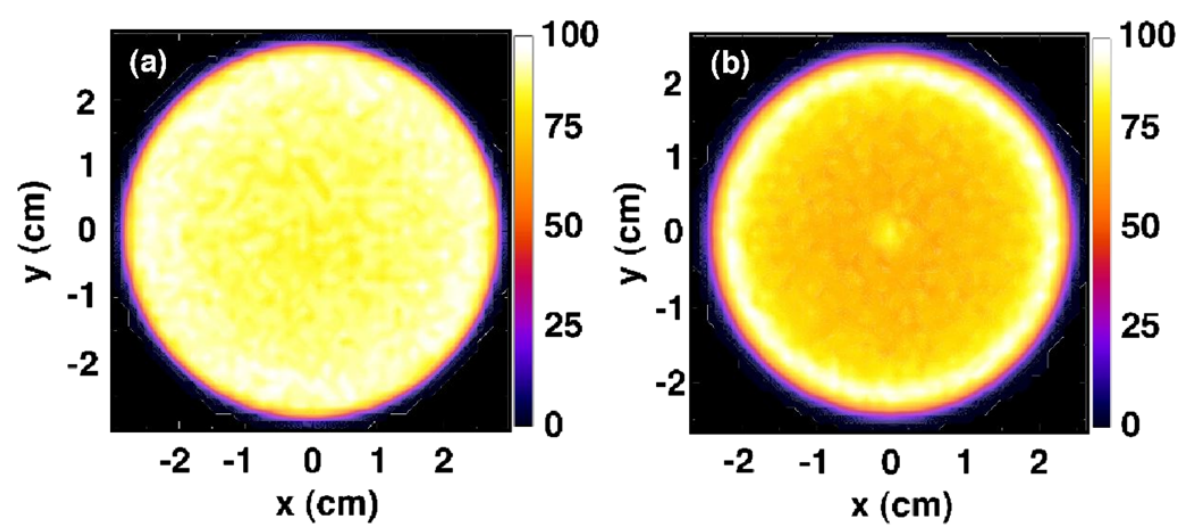

FIG. 5. (Color) Transverse beam distribution calculated at the scintillator plane $(z=43 \mathrm{~cm})$ : (a) ideally with no emitter gap; (b) with the emitter surface recessed 22 mils back from the knife edge of the Pierce cone (no focusing with solenoids; note scale differences).

Pierce cone reproduces the contoured distribution and the measured emittance with relatively good agreement [Fig. 5(b)]; there is a rim around the edge of the distribution and a slight peak in the center. This placement of the emitter surface creates a field distortion that accelerates particles at the edge into the beam, leading to the sheared distribution measured in Fig. 4(b) and calculated in Fig. 5(b).

This expected displacement of the emitter surface in these calculations is relatively small $(<1 \mathrm{~mm})$ and is difficult to measure while the emitter is under operating conditions $\left(\mathrm{T} \sim 1000^{\circ} \mathrm{C}\right)$ in vacuum. Also the geometry of the edge of the Pierce cone relative to the emitter surface, where the field distortions are created, is also difficult to measure under these operating conditions. During assembly careful attention is made to place the emitter close to the knife edge of the Pierce cone. However, adequate spacing is needed to allow for thermal expansion of the emitter housing during operation.

\section{PULSED SOLENOID}

The solenoids used for the STX were wound from litz cable consisting of 12 strands with No. 10 NEMA-35C film insulating the conductor. The cable was flat and had a rectangular cross section of $0.4 \mathrm{~cm} \times 2 \mathrm{~cm}$. Four layers with 20 turns each were wound on a 9-cm diameter, 4-mm thick NEMA G-10 tube. The magnets were potted with a layer of CTD-105 epoxy. A copper cooling water tube was added over the potted coil pack and then the assembly was potted with a heat conducting epoxy. The finished magnets were about $50-\mathrm{cm}$ long and had a mean coil radius of $5.75 \mathrm{~cm}$ and coil length of $47 \mathrm{~cm}$ [15].

Hi-pot tests were done on the conductor and breakdown voltages were in the range of $5-15 \mathrm{kV}$. These magnets are capable of producing fields up to $6 \mathrm{~T}$, but the maximum field necessary to overcome the space-charge self-field of the beam, obtain the desired envelopes, and to avoid scraping was only $3 \mathrm{~T}$. So the current drive and pulser requirements were a peak of $12 \mathrm{kA}$ over a $4 \mathrm{~ms}$ half sine pulse [15].

The magnetic fields were mapped out in detail using two B-dot probes. The probes were wound from insulated No. 32 AWG copper wire. Each probe had three axes ( $x$, $y$, and $z$ ) with 2 layers consisting of 10 turns each and a mean coil diameter of $\approx 12 \mathrm{~mm}$. One was used to map the axial magnetic field on axis [Fig. 7(a)] and the other was used to map the axial and radial magnetic fields $3.5 \mathrm{~cm}$ off 
axis. Eddy currents were also accounted for in the magnetic field measurements by using the $8.5-\mathrm{cm}$ diameter, $1.5-\mathrm{mm}$ thick beampipe, and the $2-\mathrm{cm}$ thick stainless-steel plate used as a flange at the source tank and diagnostic chamber. Measurements and simulations showed that eddy currents only decreased the focusing strength of the magnet by $1 \%[16]$.

\section{TWO-SOLENOID EXPERIMENT}

Two solenoids were placed on the NDCX beam line immediately downstream of the diode (Fig. 6). The focusing lattice consisted of two $50-\mathrm{cm}$ long solenoids spaced about $9 \mathrm{~cm}$ apart with a diagnostic box at the exit of the second solenoid. An additional cylindrical electrode was added at the exit of the last solenoid, upstream of the intercepting diagnostics, to suppress any electrons from backstreaming into the solenoid lattice. The field strengths in Fig. 7(a) were chosen to give the desired envelope shown in Fig. 7(b). If the extracted beam was ideal (zero emittance and uniform axial velocity), the solenoid field of $2.5 \mathrm{~T}$ used to match the space-charge self-field of the beam, $13 \mathrm{~mm}$ in radius, would establish Brillouin flow at a Larmor frequency $\left(\omega_{c} / 2\right)$ of $3 \times 106 \mathrm{rad} / \mathrm{sec}$.

Note there is a small fringe magnetic field of $0.3 \mathrm{kG}$ at the emitter surface and a significant fringe field of $1 \mathrm{kG}$ at $z=142 \mathrm{~cm}$ (upstream slit location), $11 \mathrm{~cm}$ downstream of the exit of two solenoids. The field at the emitter surface can contribute to a small canonical angular momentum that may cause hollowness in the beam distribution after focusing [17]. Calculations show the canonical angular momentum defocusing term is smaller than the emittance defocusing term in the envelope equation and WARP calculations confirm it is negligible.

\section{A. Observed electron cloud effects}

Electron cloud dynamics differ significantly in beam transport with solenoids compared to magnetic quadrupoles. Magnetic quadrupoles do not have a strong axial magnetic field but rather a strong transverse magnetic field (or strong gradient in the axial direction). This provides for a more rapid falloff of the field in the axial direction compared to solenoids. Initial measurements of the beam at $z=142 \mathrm{~cm}, 11 \mathrm{~cm}$ downstream of the exit of the solenoid lattice, showed unexpected emittance growth and time dependence. For example, transverse phase-space measurements $1 \mu \mathrm{s}$ after the beam head displayed an emittance increase of about a $60 \%$ from that measured at the gun (Figs. 3 and 8). The emittance continued to rise throughout the pulse and time dependence was observed in the beam envelope (Fig. 8).

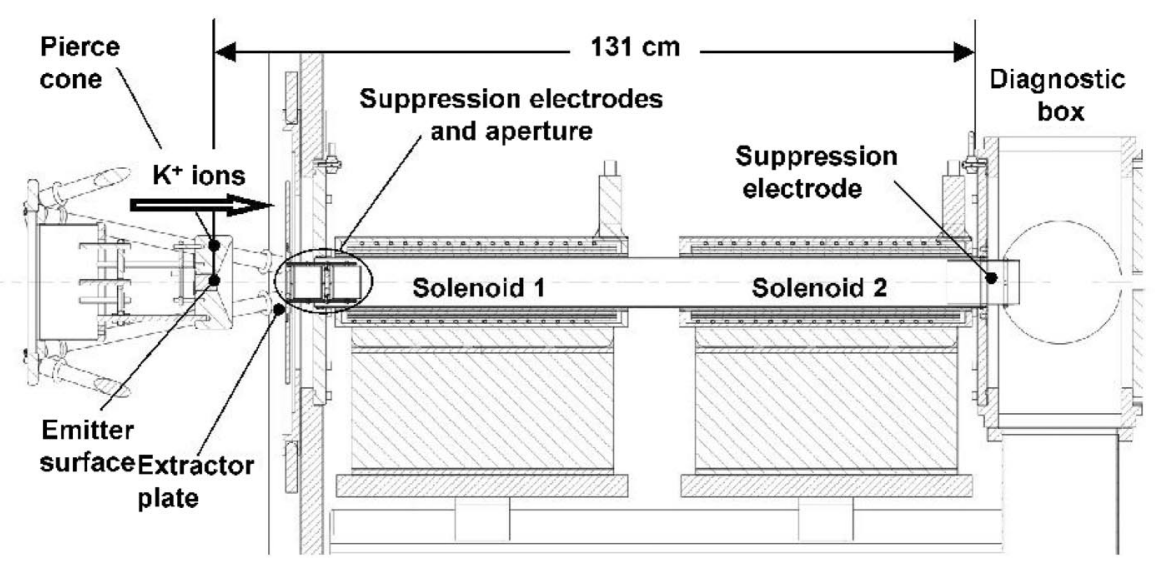

FIG. 6. Elevation view of the two-solenoid experiment.
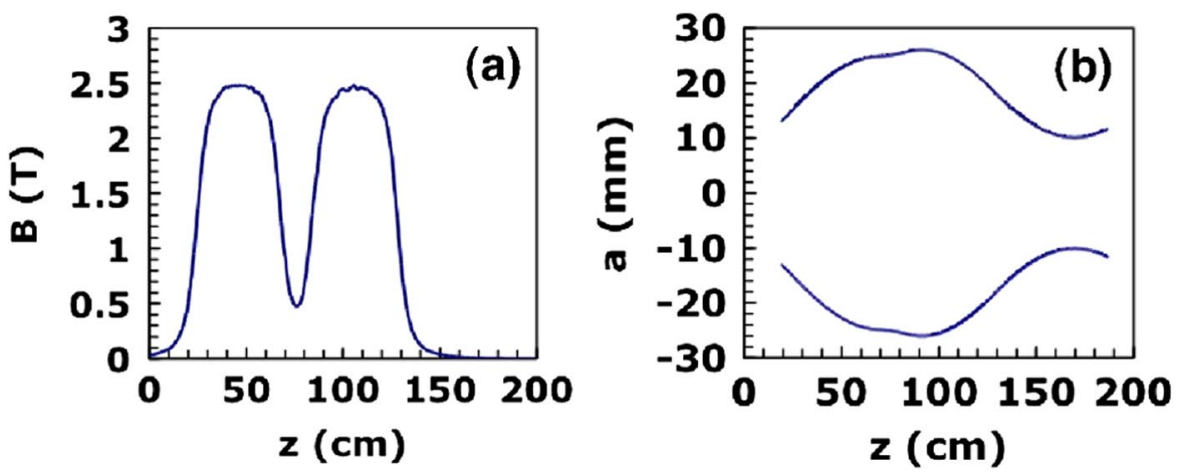

FIG. 7. (Color) (a) Axial magnetic field profile based on measurements; (b) calculated beam envelope. 

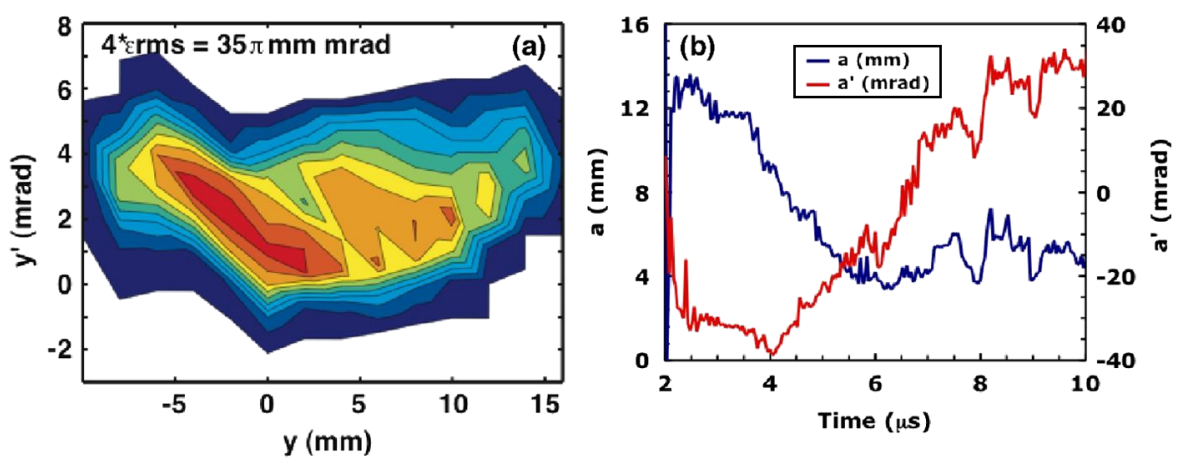

FIG. 8. (Color) (a) Sheared phase-space distribution integrated over a 500 ns gate $1 \mu$ s into beam pulse; (b) beam envelope as a function of time $11 \mathrm{~cm}(z=142 \mathrm{~cm})$ downstream of the exit of two solenoids.

An unusual signal was also measured on the suppression electrode at the exit of the second solenoid when a slit paddle intercepted the beam. There was a positive capacitive image current at about $1 \mu \mathrm{s}$ when the beam entered the diagnostic followed by a rising positive current and then high-frequency oscillations with a period of about $100 \mathrm{~ns}$ [Fig. 9(a)]. Details of the oscillations were found to be random; every shot showed a somewhat different highfrequency pattern. A decrease in the beam radius and a change in the envelope angle were measured at the onset of these high-frequency oscillations [Fig. 8(b)]. Over the $10-\mu \mathrm{s}$ pulse the beam envelope angle switched from a converging angle of $30 \mathrm{mrad}$ to a diverging angle of $30 \mathrm{mrad}$, suggesting that the beam was being neutralized upstream due to backstreaming electrons that were not effectively suppressed by the suppression electrode regardless of the bias voltage.

It is known that intense ion beams will yield electrons from secondary emission and gas desorption and ionization processes once the beam hits an intercepting diagnostic [18-21]. The slit paddle that intercepts the beam path is composed of stainless steel, which is known to adsorb hydrogen. In another experiment with a higher kinetic energy beam, residual gas measurements during the pres- sure rise from a $\mathrm{K}^{+}$beam incident on stainless steel show that hydrogen is the main component of the desorbed gas and it expands into the beam path with an average velocity of $0.5 \mathrm{~mm} / \mu \mathrm{s}$ [22].

Particle in the cell simulations using the WARP code [14] were conducted in order to reproduce and better understand the interactions of the beam with desorbed gas, and subsequent ions and electrons [23]. These interactions are also relevant to electron cloud studies in other high intensity accelerators [24-33]. The calculated current on the suppression electrode [Fig. 9(b)] has several features that qualitatively agree with the experimental measurement. A positive capacitive image current appears at about $1 \mu \mathrm{s}$ when the beam enters the diagnostic. The calculated time for a positive current to appear on the suppressor due to ionized $\mathrm{H}_{2}$ is about $1 \mu \mathrm{s}$ after the head of the beam, consistent with the observations in this experiment. This positive current continues to rise and then is followed by high-frequency oscillations.

The observed high-frequency oscillations on the suppression electrode are due to an oscillating virtual cathode [34]. Once enough desorbed hydrogen gas is ionized the $\mathrm{H}_{2}{ }^{+}$and electron densities immediately in front of the slit plate increase to about the beam density, enough to over-
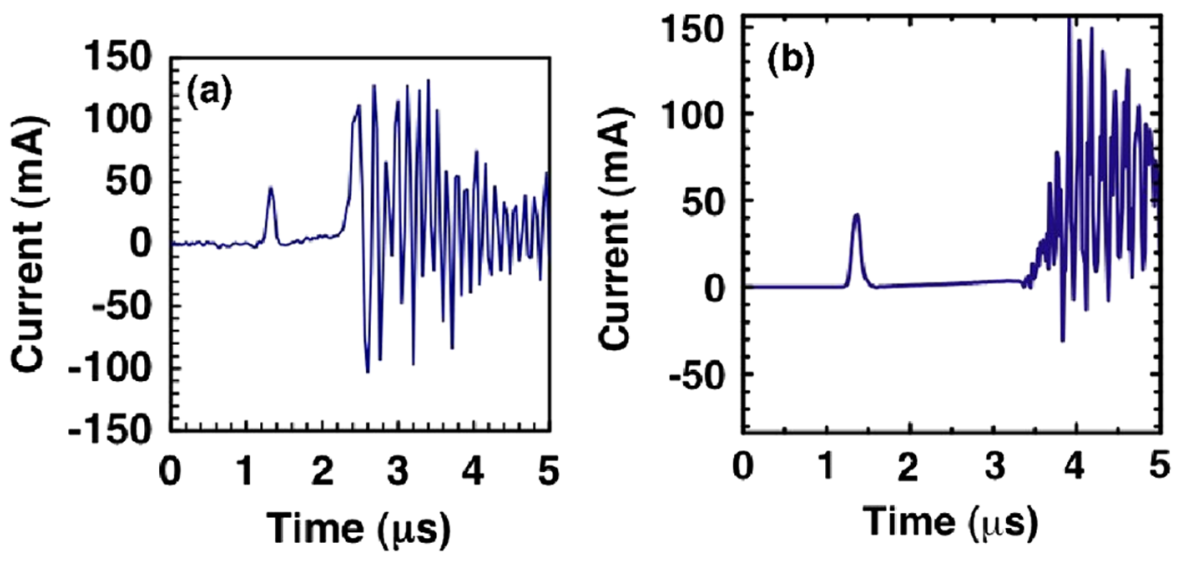

FIG. 9. (Color) (a) Signal measured on the suppression electrode at the exit of the second solenoid with a slit paddle intercepting the beam; (b) current calculated for the same case by a WARP simulation (note scale differences). 

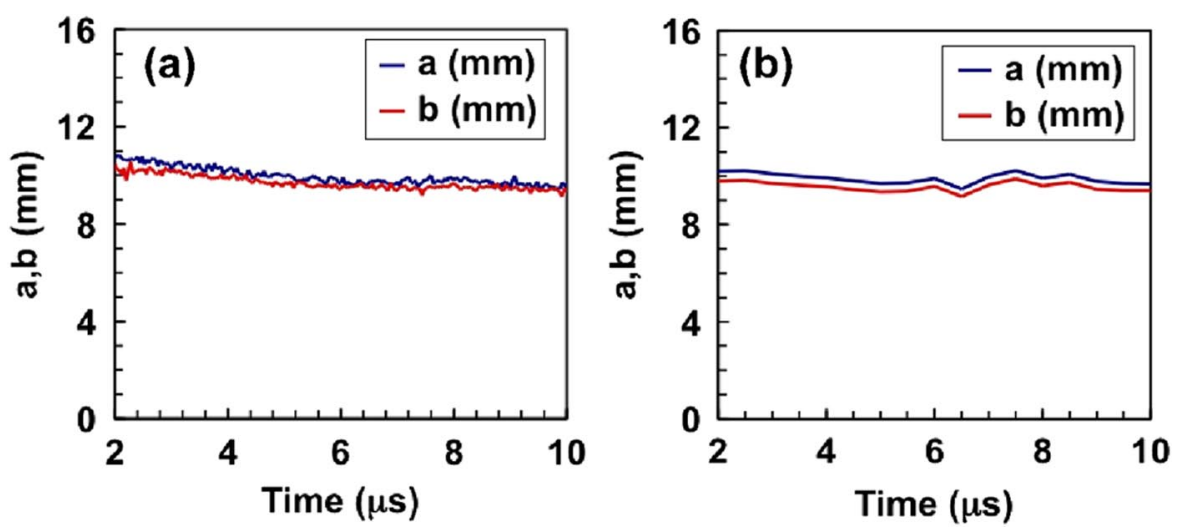

FIG. 10. (Color) Beam radii: (a) $21 \mathrm{~cm}(z=152 \mathrm{~cm})$ and; (b) $27 \mathrm{~cm}(z=158 \mathrm{~cm})$ downstream of the exit of two solenoids as a function of time.

come the suppressor potential. Each species forms a sheath and they oscillate at the electron plasma frequency. This process pushes electrons through the suppressor and $3 \mu \mathrm{s}$ after the head of the beam a sufficient amount of electrons backstream into the solenoid fields to partially neutralize the beam. Beam measurements are consistent with this showing an increase in emittance and a gradual decrease in the beam radius at $4 \mu$ s until a $60 \%$ reduction is reached around $5 \mu \mathrm{s}$ and the beam begins to diverge [Fig. 8(b)].

Through more detailed observations, it was determined that the deviations in the measured envelope parameters throughout the beam pulse only occur when the upstream slit paddle intercepts the beam. When the slit cup and scintillator diagnostics, 10 and $16 \mathrm{~cm}$ further downstream, were used much weaker time dependence was observed in the beam radius (Fig. 10). As noted in Sec. II, this envelope fluctuation was not observed when the beam was diagnosed upstream at the gun without any focusing fields. These observations hint electron confinement by the large fringe magnetic fields $(1-1.3 \mathrm{kG})$ helped contribute to the sheath formation and electron cloud and gas effects de- scribed above, confounding the measurement of the intrinsic beam distribution.

\section{B. Mitigation of electron cloud effects}

A drift distance of $29 \mathrm{~cm}$ was added between the end of the second solenoid and the suppressing and intercepting diagnostics to test whether measuring the beam distribution with an intercepting diagnostic in a strong magnetic field will confuse the measurement. Each of the diagnostics was now immersed in a field strength nearly an order of magnitude less. This also increased the gyroradii of electrons to several $\mathrm{cm}$, which was on the order of the diameter of the suppression electrodes.

Time dependent measurements of the transverse phase space showed the measured emittance was reduced from previous measurements, and close to the injected beam emittance $(22 \pi \mathrm{mm}$ mrad, Fig. 11). These measurements also demonstrated excellent agreement between the two methods of measuring the phase space; double slit and a Faraday collector and optical measurements. The measured beam envelope (at $z=171 \mathrm{~cm}$ ) also no longer varied
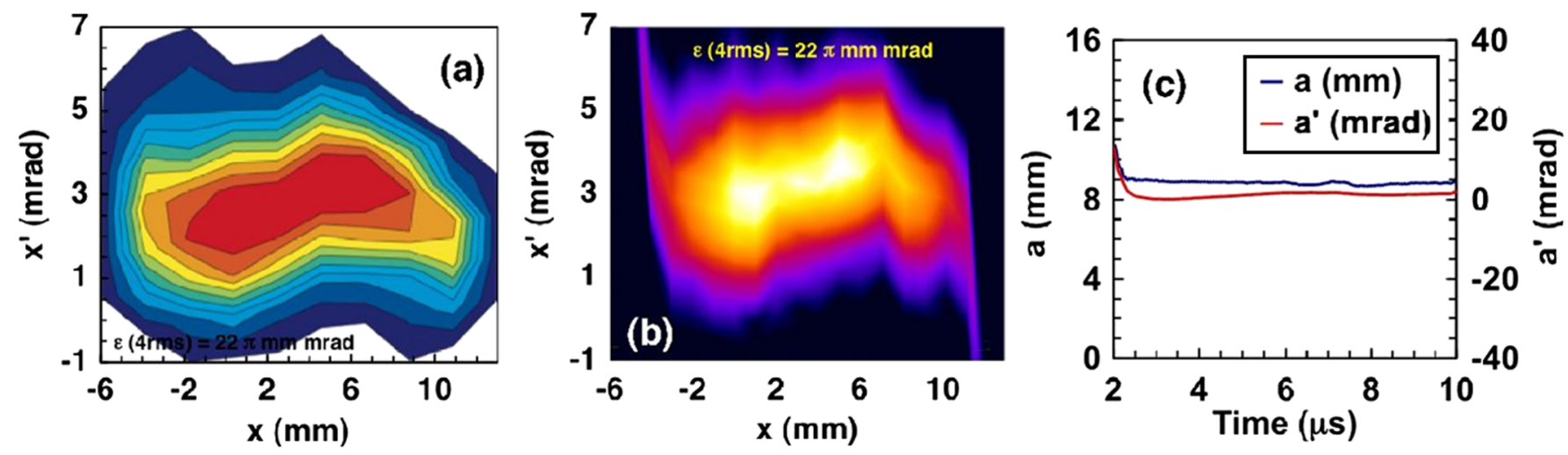

FIG. 11. (Color) Sheared phase-space distribution integrated over a 500 ns gate in the middle of the $10 \mu$ s beam pulse: (a) measured with a double slit and a Faraday collector; (b) measured optically and; (c) beam envelope as a function of time. All were measured $40 \mathrm{~cm}$ downstream of the exit of two solenoids $(z=171 \mathrm{~cm})$. 

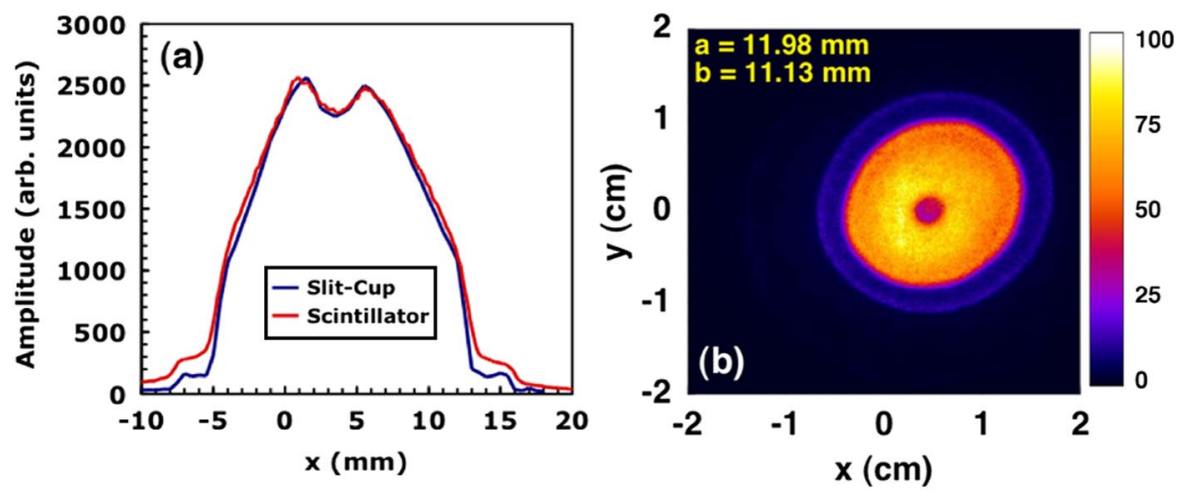

FIG. 12. (Color) Transverse profile of the beam measured: (a) $50 \mathrm{~cm}(z=181 \mathrm{~cm})$ and $56 \mathrm{~cm}(z=187 \mathrm{~cm})$ downstream of the exit of two solenoids. (b) Transverse beam distribution at the scintillator plane. All integrated over a $500 \mathrm{~ns}$ gate in the middle of the $10 \mu \mathrm{s}$ beam pulse.

drastically in time [Fig. 11(c)] and agreed well with the calculated envelope shown in Fig. 7(b).

Time resolved measurements of the transverse beam distribution and profile complemented the phase-space measurements by also displaying a constant radius versus time, showing that spurious electron cloud effects due to the intercepting diagnostics were mitigated as required for WDM and fusion applications. Despite the nearly uniform and steady-state envelope, the transverse beam distribution and profile were not uniform or axisymmetric (Fig. 12). The beam centroid was offset by several millimeters and milliradians, had a hollow center, and a substantial halo. The causes for these undesired effects in the beam distribution were not well understood and were explored in the WARP code.

\section{Alignment effects on beam dynamics}

Particle in the cell simulations with the WARP code [14] were used to quantify and reproduce the features of the distribution measured at the exit of the transport lattice. As shown in Sec. II recessing the emitter surface 22 mils back from the knife edge of the Pierce cone reproduces the contoured distribution with relatively good agreement; there is a rim around the edge of the distribution, and the slight peak in the center [Fig. 5(b)]. This placement of the emitter surface creates a field distortion that accelerates particles at the edge into the beam leading to the sheared distribution measured in Fig. 4(b) and calculated in Fig. 5(b).

Calculations with WARP indicate that transporting this sheared distribution through a perfectly aligned solenoid lattice reproduces some of the features in the measured distribution in Fig. 12(b). Using the recessed emitter alone reproduced the radial contours and the density depression in the center of the distribution (Fig. 13); however, the distribution was still well centered and axisymmetric.

The centroid offsets of the beam distribution are only reproduced after adding solenoid misalignments to the WARP calculation [Fig. 14(a)]. It is known from mapping the fields of the solenoids that there are single millimeter offsets of the coils within the magnet structure. Precisely adding the actual solenoid misalignments is a difficult task, which requires a detailed set of data. Each solenoid has four individual displacements $\left(\langle x\rangle,\left\langle x^{\prime}\right\rangle,\langle y\rangle\right.$, and $\left.\left\langle y^{\prime}\right\rangle\right)$, two position and two angular displacements relative to the ideal beam axis.

A measurement of the 4D phase space of the beam provided four individual centroid offsets $\left(\langle x\rangle,\left\langle x^{\prime}\right\rangle,\langle y\rangle\right.$, and $\left\langle y^{\prime}\right\rangle$ ) that were inverted to solve for the $x$ and $y$ position displacements of the two solenoids only (Table I). This approximation was made to determine the impact of the solenoid position offsets on the beam distribution. The solenoid displacements determined from this inversion were quite large due to the simplicity of the model (i.e. it excluded angular offsets and initial offsets). It has been

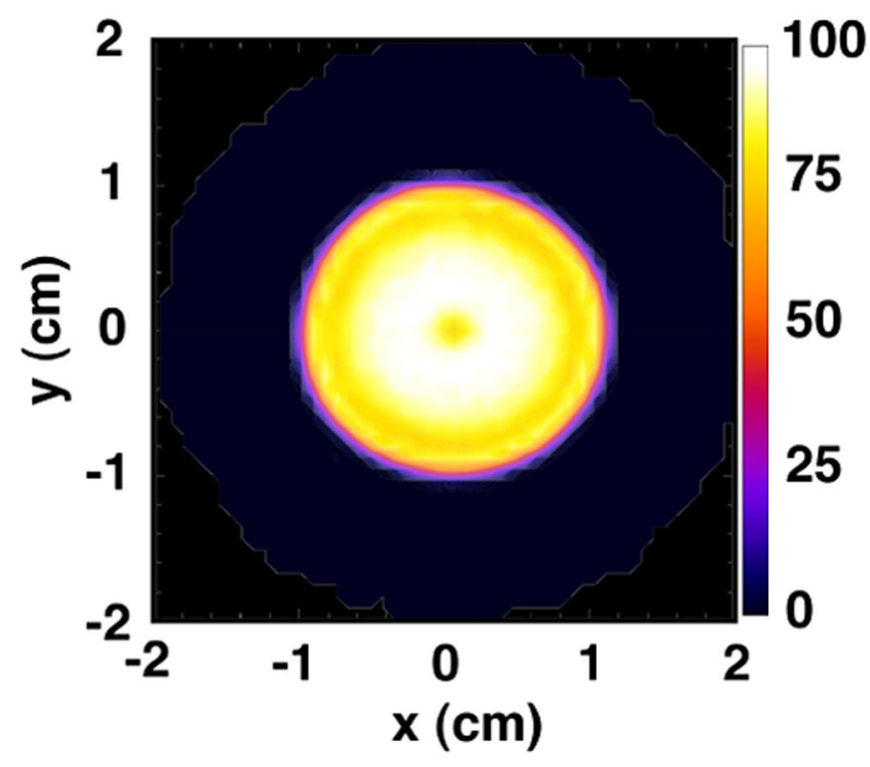

FIG. 13. (Color) Transverse beam distribution calculated $56 \mathrm{~cm}$ downstream of the exit of two solenoids at the scintillator plane $(z=187 \mathrm{~cm})$ using the recessed emitter. 

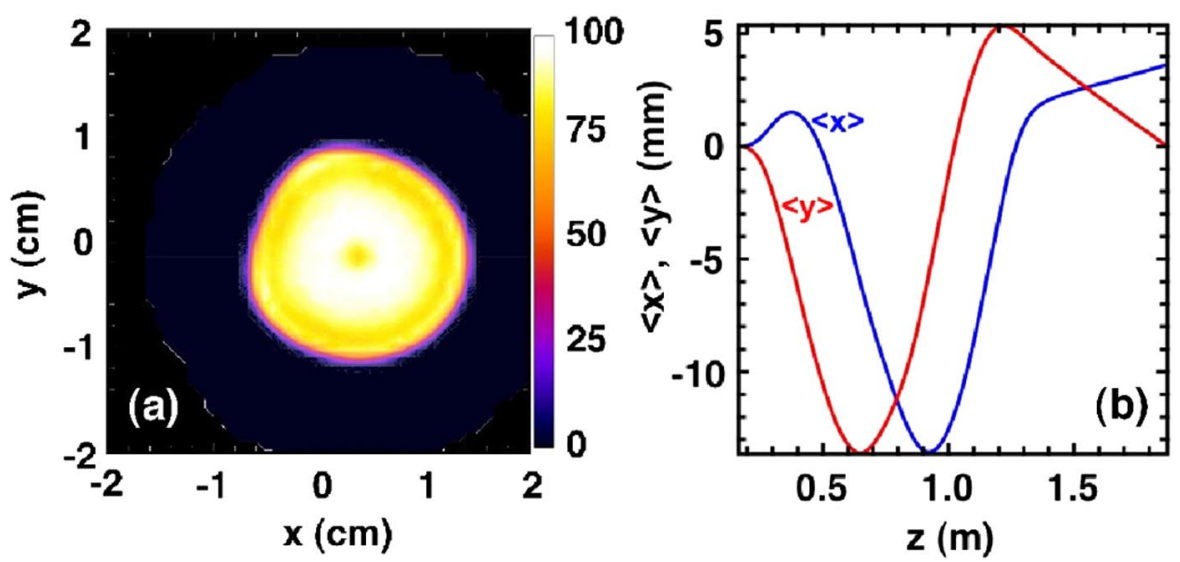

FIG. 14. (Color) (a) Transverse beam distribution calculated $56 \mathrm{~cm}$ downstream of the exit of two solenoids at the scintillator plane $(z=187 \mathrm{~cm})$ using the recessed emitter and misaligned solenoids; (b) beam centroid offsets along the axis of propagation.

TABLE I. Approximation of $x$ and $y$ displacements of the two solenoids relative to the ideal beam axis.

\begin{tabular}{lrr}
\hline \hline & $\mathrm{S} 1$ & \multicolumn{1}{c}{$\mathrm{S} 2$} \\
\hline$\Delta x(\mathrm{~mm})$ & -15.88 & -0.77 \\
$\Delta y(\mathrm{~mm})$ & -9.90 & -15.80 \\
\hline \hline
\end{tabular}

determined analytically that small angular displacements $(<5 \mathrm{mrad})$ of the solenoids can contribute to equal or greater beam centroid offsets when compared with solenoid position displacements of $<3 \mathrm{~mm}$. A position offset $<3 \mathrm{~mm}$ is equivalent to a angular displacement $<10 \mathrm{mrad}$ about the center of a $50-\mathrm{cm}$ long solenoid.

These calculations were able to reproduce the measured centroid offsets of beam in Figs. 11 and 12 reasonably well [Fig. 14(a)] and maintain the contoured distribution with the density depression in the center. The resulting betatron motion of the beam centroid due to the solenoid offsets is shown in Fig. 14(b).
Offsetting the solenoids is an arbitrary option in the WARP calculation. The same effect could be demonstrated by offsetting the beam injected into the solenoid lattice. However, from the measurements in Sec. II it is evident that the extracted beam is well aligned. It would take an offset comparable to the solenoid offsets to demonstrate the observed centroid offsets.

Up to this point we have been able to produce a contoured distribution with a density depression in the center and centroid offsets by adding a recessed emitter and solenoid offsets to the WARP calculation. However, the calculated distribution in Fig. 14(a) does not display the nonaxisymmetric or elliptical shape measured in Fig. 12(b). Since the beam is suffering from betatron motion of its centroid, it is sampling a greater portion of the nonlinear fields than a perfectly centered beam distribution. This will affect the shape of the beam distribution and it must be included in the calculation.

The fringe components of a focusing element are the most significant contributor to nonlinear focusing effects. Consider a finite length current sheet wrapped around the
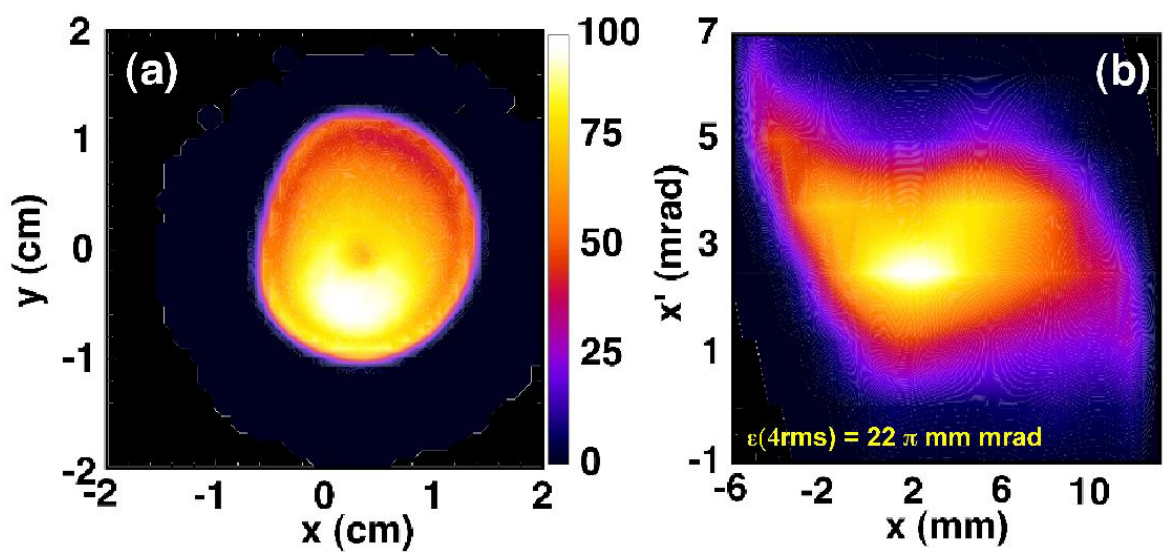

FIG. 15. (Color) (a) Transverse beam distribution calculated $56 \mathrm{~cm}$ downstream of the exit of two solenoids at the scintillator plane $(z=187 \mathrm{~cm})$; (b) sheared phase-space distribution calculated $40 \mathrm{~cm}$ downstream of the exit of two solenoids at the scintillator plane $(z=171 \mathrm{~cm})$. All were calculated using the recessed emitter, misaligned solenoids, and nonlinear focusing terms. 
$z$-axis (in the azimuthal direction). This current sheet acts as an ideal solenoid creating an ideal magnetic field which is linear except near the ends where the field begins to fringe out. Using this approximation, one can understand how the fringe fields of a solenoid are the largest contributors to nonlinear focusing.

After adding the nonlinear field terms into the calculation a better qualitative agreement is observed between the measured distribution in Fig. 12(b) and the calculation [Fig. 15(a)]. An elliptical shape is observed in addition to a density peak in the lower left of the distribution. The sheared phase space and emittance calculated in Fig. 15(b) is also in good qualitative agreement with those measured in Figs. 11(a) and 11(b). The centroid offsets are identical by calculation and the distortions at the edge have also been reproduced.

Although the details of the distributions were not exactly reproduced, the factors that contributed to the features seen in the distribution are understood. The recessed emitter causes a nonuniform distribution to be injected into the solenoid lattice and the misaligned solenoids cause a centroid offset to evolve. The shift in the charge distribution [Fig. 12(b) and 15(a)] is due to nonlinear focusing of the beam.

Precise alignment of the axial magnetic field in a solenoid lattice is critical to the beam dynamics. Slight misalignment of the solenoids in a focusing lattice causes the beam centroid to carry out a corkscrew orbit and this motion can grow axially along a focusing lattice if each additional solenoid is misaligned [35,36]. This excitation can also lead to emittance growth and halo formation [37]. These undesired effects have been studied on several electron beam experiments and can become catastrophic as the number of lattice elements increases. Steering dipoles have been used in the past and will also be used here to correct the centroid motion $[38,39]$.

\section{CONCLUSIONS}

We have successfully demonstrated matching and transport of a space-charge dominated ion beam in a twosolenoid lattice with little or no emittance growth. It is evident from the results presented that large fringe magnetic fields help electron confinement and contribute to the sheath formation and electron cloud effects observed, confusing measurements. Moving the beam intercepting diagnostics into a nearly magnetic field-free region provided the correct measurement of the beam dynamics and the emittance without impacting the beam.

Precise placement of the emitter and alignment of the axial magnetic field in a solenoid lattice is critical to the beam dynamics. Evidence of this importance is seen in the measured and calculated beam distributions which are not uniform or axisymmetric, have a centroid offset of several millimeters and milliradians, a hollow center, and a substantial halo. Although these undesired effects have little impact on the emittance in this two-solenoid experiment, they may grow in longer focusing lattices and contribute to emittance growth or beam loss.

\section{ACKNOWLEDGMENTS}

This work was supported by the Director, Office of Science, Office of Fusion Energy Sciences, of the U.S. Department of Energy under Contract No. DE-AC0205CH11231. Special thanks go to Tak Katayanagi, Wayne Greenway, Craig Rogers, Ed Romero, and Andy Faltens for their continued technical support.

[1] C. F. Chan et al., Nucl. Instrum. Methods Phys. Res., Sect. A 415, 274 (1998).

[2] E. Lee, Nucl. Instrum. Methods Phys. Res., Sect. A 544, 187 (2005).

[3] H.V. Smith et al., in Proceedings of the Particle Accelerator Conference, New York, NY, 1999, p. 1929.

[4] A. Ueno et al., in Proceedings of the XX International Linac Conference, Monterey, CA, 1999, p. 256.

[5] A. Jakob et al., in Proceedings of the European Particle Accelerator Conference, Paris, France, 2002, p. 1906, http://www.JACoW.org/.

[6] E. Fagotti et al., in Proceedings of the 2004 International Linac Conference, Lübeck, Germany, 2004, p. 72.

[7] M. A. Leitner et al., Nucl. Instrum. Methods Phys. Res., Sect. A 544, 315 (2005).

[8] B. G. Logan et al., in Proceedings of the Workshop on Accelerator-Driven High Energy Density Physics, LBNL, 2004, Appendix A1 (LBNL-57518, UCRLPROC-212000, 2005), http://hifweb.lbl.gov/public/ hedpworkshop/toc.html

[9] P. K. Roy et al., Phys. Rev. Lett. 95, 234801 (2005).

[10] P. A. Seidl et al., Nucl. Instrum. Methods Phys. Res., Sect. A 577, 215 (2007).

[11] J. P. Blewett and E. J. Jones, Phys. Rev. 50, 464 (1936).

[12] A. I. Warwick, in Proceedings of the Particle Accelerator Conference, Vancouver, Canada, 1985, p. 1809, http:// www.JACoW.org/.

[13] F. M. Bieniosek et al., Nucl. Instrum. Methods Phys. Res., Sect. A 544, 268 (2005).

[14] D. P. Grote et al., AIP Conf. Proc. 749, 55 (2005).

[15] D. Shuman et al., in Proceedings of the Particle Accelerator Conference, Knoxville, Tennessee, 2005, p. 3798, http://www.JACoW.org/.

[16] J. Armijo, LBNL Report No. 61975, 2006.

[17] Martin Reiser, Theory and Design of Charged Particle Beams (John Wiley and Sons Inc., New York, 1994), pp. 210 and 318.

[18] A.W. Molvik et al., Phys. Rev. ST Accel. Beams 7, 093202 (2004).

[19] M. Kireeff Covo et al., Phys. Rev. ST Accel. Beams 9, 063201 (2006).

[20] M. Kireeff Covo et al., Phys. Rev. Lett. 97, 054801 (2006).

[21] A. W. Molvik et al., Phys. Rev. Lett. 98, 064801 (2007).

[22] F. M. Bieniosek et al., Phys. Rev. ST Accel. Beams 10, 093201 (2007). 
[23] W. M. Sharp et al., Nucl. Instrum. Methods Phys. Res., Sect. A 577, 146 (2007).

[24] G. Budker, G. Dimov, and V. Dudnikov, Sov. At. Energy 22, 5 (1967).

[25] O. Gröbner, in Proceedings of the 10th International Conference on High-Energy Accelerators, Protvino, Russia (Institute of High Energy Physics, Protvino, 1977), p. 277.

[26] M. Izawa, Y. Sato, and T. Toyomasu, Phys. Rev. Lett. 74, 5044 (1995).

[27] K. Ohmi, Phys. Rev. Lett. 75, 1526 (1995).

[28] W. T. Weng et al., in Proceedings of the Particle Accelerator Conference, Vancouver, Canada, 1997, p. 970, http://www.JACoW.org/.

[29] R. J. Macek et al., in Proceedings of the Particle Accelerator Conference, Chicago, Illinois, 2001, p. 688, http://www.JACoW.org/.

[30] M. A. Furman, LBNL Report No. 50765, 2002.

[31] S. Y. Zhang et al., in Proceedings of the European Particle
Accelerator Conference, Lucerne, Switzerland, 2004, p. 947, http://www.JACoW.org/.

[32] J. W. Flanagan et al., Phys. Rev. Lett. 94, 054801 (2005).

[33] R. J. Macek and A. A. Browman, in Proceedings of the Particle Accelerator Conference, Knoxville, Tennessee, 2005, p. 2547, http://www.JACoW.org/.

[34] W. Jiang and Magne Kristiansen, Phys. Plasmas 8, 3781 (2001).

[35] Y.-J. Chen et al., Nucl. Instrum. Methods Phys. Res., Sect. A 292, 455 (1990).

[36] Y.-J. Chen, Nucl. Instrum. Methods Phys. Res., Sect. A 398, 139 (1997).

[37] Y.-J. Chen et al., in Proceedings of the Particle Accelerator Conference, San Francisco, CA, 1991, p. 3100.

[38] Y.-J. Chen et al., in Proceedings of the XIX International Linac Conference, Chicago, IL, 1998, p. 469.

[39] T. L. Houck et al., in Proceedings of the XIX International Linac Conference, Chicago, IL, 1998, p. 857. 